Supporting Information

\title{
Multispectroscopic and Molecular Docking Insight into Elucidating the Interaction of Irisin with Rivastigmine Tartrate: A Combinational Therapy Approach to Fight Alzheimer's Disease
}

Rashid Waseem ${ }^{1}$, Anas Shamsi ${ }^{1}$, Taj Mohammad ${ }^{1}$, Fahad A Alhumaydhi ${ }^{2}$, Syed Naqui Kazim ${ }^{1}$, Md. Imtaiyaz Hassan ${ }^{1}$, Faizan Ahmad ${ }^{1}$, Asimul Islam ${ }^{1 *}$

${ }^{I}$ Centre for Interdisciplinary Research in Basic Sciences, Jamia Millia Islamia, New Delhi.

${ }^{2}$ Department of Medical Laboratories, College of Applied Medical Sciences, Qassim University, Buraydah 52571, Saudi Arabia.

*Corresponding Author:

Dr. Asimul Islam, M.N.A.Sc.

Associate Professor

Centre for Interdisciplinary Research in Basic Sciences; Jamia Millia Islamia, New Delhi;

Email: aislam@jmi.ac.in

Ph.: +91-9312812007 


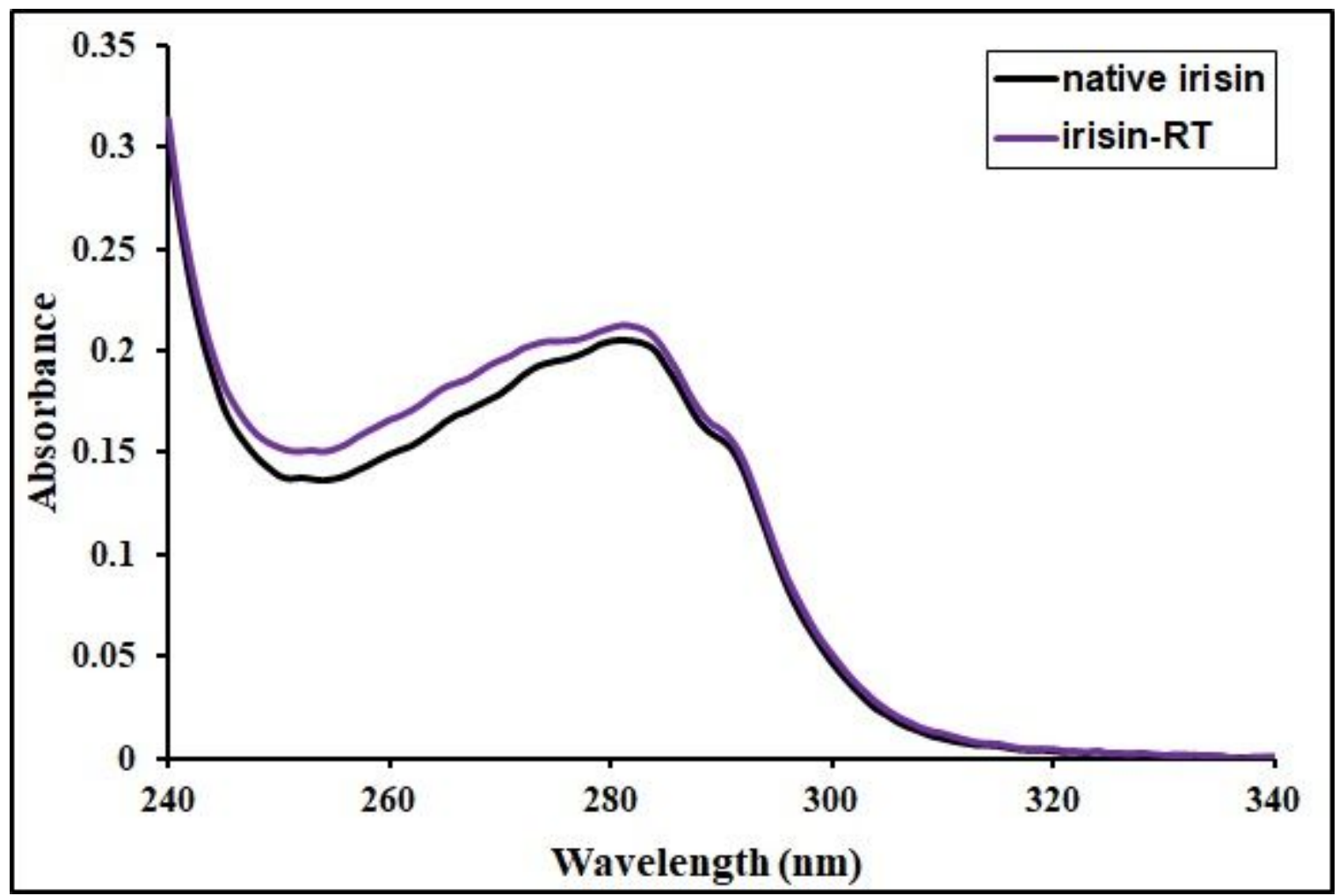

Figure SI: UV spectra of irisin in the absence and presence of $R T(1: 9)$. 